\title{
Prothrombotic clot properties can predict venous ulcers in patients following deep vein thrombosis: a cohort study
}

\author{
Maciej Wiktor Polak ${ }^{1} \cdot J_{a k u b}$ Siudut ${ }^{1,2} \cdot$ Krzysztof Plens $^{3} \cdot$ Anetta Undas $^{1,2}$ (D)
}

Published online: 20 August 2019

(c) The Author(s) 2019

\begin{abstract}
Venous ulcers are the most severe manifestation of post-thrombotic syndrome (PTS). We have previously demonstrated that formation of compact fibrin clots resistant to lysis is observed in patients following deep-vein thrombosis (DVT) who developed PTS. The current study investigated whether unfavourable fibrin clot properties can predict post-thrombotic venous ulcers. In a cohort study on 186 consecutive patients following DVT, we determined plasma fibrin clot characteristics, including clot permeability and lysability, inflammatory markers, thrombin generation, fibrinolysis proteins at 3 months since the index event. Occurrence of PTS and venous ulcers was recorded during follow-up (median, 53; range 24 to 76 months). Fifty-seven DVT patients (30.6\%) developed PTS, including 12 subjects (6.45\%) with a venous ulcer (4 individuals with recurrent ulcers). Patients who developed ulcers compared with the remainder had at enrolment $13.0 \%$ lower clot permeability $\left(\mathrm{K}_{\mathrm{s}}\right), 17.4 \%$ longer clot lysis time (CLT), $13.1 \%$ longer lag phase of clot formation, and 5.0\% higher maximum absorbance, with no difference in fibrinogen, C-reactive protein, and thrombin generation. The baseline prothrombotic fibrin clot phenotype $\left(\mathrm{K}_{\mathrm{s}} \leq 6.5 \times 10^{-9} \mathrm{~cm}^{2}\right.$ and CLT $\left.>100 \mathrm{~min}\right)$ was associated with a higher risk of ulcers [hazard ratio (HR), 5.37; 95\% confidence interval (CI), 1.3-21.5]. A multivariate model adjusted for age, sex, and fibrinogen showed that independent predictors of the ulcer occurrence were body mass index (HR 1.53; 95\% CI 1.30-1.86), CLT (HR 1.43; 95\% CI 1.04-2.05), and $\alpha_{2}$-antiplasmin (HR 0.95; 95\% CI 0.90-0.99). This study suggests that formation of denser fibrin clots with impaired fibrinolysis predisposes to post-thrombotic venous ulcers.
\end{abstract}

Keywords Venous ulcer $\cdot$ Post-thrombotic syndrome $\cdot$ Fibrin $\cdot$ Fibrinolysis $\cdot$ Thrombosis

\section{Highlights}

- Post-thrombotic syndrome (PTS) is a common sequela of deep-vein thrombosis (DVT).

Electronic supplementary material The online version of this article (https://doi.org/10.1007/s11239-019-01914-w) contains supplementary material, which is available to authorized users.

Anetta Undas

mmundas@cyf-kr.edu.pl

1 Institute of Cardiology, Jagiellonian University Medical College, 80 Pradnicka St, 31-202 Krakow, Poland

2 Krakow Centre for Medical Research and Technologies, John Paul II Hospital, Krakow, Poland

3 KCRI, Krakow, Poland
- Prothrombotic clot properties, including dense fibrin networks resistant to lysis, have been observed in patients with DVT who developed PTS during follow-up.

- The most severe manifestation of PTS is a venous ulcer. The known risk factors of venous ulcer formation are older age, obesity and the presence of reflux in deep veins of the lower extremity.

- Patients who develop venous ulcers during follow-up tend to display a specific fibrin clot phenotype, including reduced clot permeability and susceptibility to lysis, at 3 months since the index DVT.

- Prolonged clot lysis time (CLT), lower $\alpha_{2}$-antiplasmin and elevated body mass index were identified as independent predictors of the post-thrombotic venous ulcer. It can be speculated that measurement of clot permeability and CLT might be useful in identifying DVT patients at high risk of post-thrombotic ulcers. 


\section{Introduction}

A venous ulcer is a debilitating manifestation of severe postthrombotic syndrome (PTS) following deep-vein thrombosis (DVT). PTS develops in $20 \%$ to $50 \%$ of DVT patients, and severe PTS, including venous ulcers, develops in 5\% to $10 \%$ [1]. The prevalence of venous ulcers has been estimated to range between 0.1 and $2 \%[2,3]$. Management of venous ulcers is a challenge for a patient and healthcare system [4], given their recurrences in 26-69\% of patients within 12 months [5].

Venous ulcers tend to form in the gaiter area of the leg, shallow, surrounded by oedematous tissue and dilated veins [6]. The venous leg ulcer has been postulated to arise from a chronic inflammatory injury secondary to sustained venous hypertension [7]. The development of venous ulcers might result from primary venous valvular reflux or be secondary to previous DVT event [4].

The principal risk factors for PTS are anatomically extensive DVT, failure of vein recanalization, recurrent ipsilateral DVT, persistent leg symptoms 1 month after acute DVT, obesity, and older age [1]. Risk factors for ulcers involve age [8], presence of the reflux in the deep veins [9], high body mass index (BMI), and low physical activity [10], as well as potentially male sex, smoking, diabetes, peripheral arterial disease and leg injury [11]. Anticoagulant therapy and use of compression stockings do not decrease the risk of ulcer development [12].

Blood coagulation eventually results in formation of fibrin clots and their characteristics, reflected by its density and lysability, differ significantly among individuals in association with various genetic and environmental factors [13]. It has been shown that clots composed of thin and highly branched fibrin fibres predispose to arterial and venous thromboembolic events and might have a predictive value in terms of recurrent episodes [14-16]. In 2016 we found that the prothrombotic plasma clot phenotype detected about 3 months since acute DVT is associated with PTS during follow-up [17]. More compact fibrin clot phenotype was also associated with higher plasma D-dimer, C-reactive protein (CRP) and thrombin-activatable fibrinolysis inhibitor (TAFI). However, none of those patients had venous ulcers.

We hypothesised that the unfavourable fibrin clot phenotype increases the risk of developing venous ulcers in DVT patients. For this reason, we performed a cohort study among DVT patients with a long-term follow-up to assess association between fibrin properties and venous ulcers.

\section{Patients and methods}

We studied 197 patients with documented first-ever DVT. Patient characteristics were presented in detail previously [17]. They were referred to an outpatient clinic for further diagnostic work-up between October 2008 and June
2010. The diagnosis of DVT was established by a positive finding on colour duplex sonography. The diagnosis of pulmonary embolism was based on the presence of typical symptoms and positive results of high-resolution spiral computed tomography. A venous thromboembolism (VTE) episode was defined as unprovoked if the patient had no history of cancer, surgery requiring general anaesthesia, major trauma, a plaster cast or hospitalization within the last month, or pregnancy or delivery within the last 3 months. Patients were treated with heparin followed by vitamin $\mathrm{K}$ antagonist (VKA).

\section{Laboratory investigations}

Blood samples were collected after fasting from individuals after about 3 months of anticoagulant therapy following the DVT diagnosis. Prior to the testing, patients receiving VKA treatment were switched to heparin for 10-14 days. $12 \mathrm{~h}$ following the last injection, when the INR was $<1.2$, samples were drawn. Detailed methodology was presented previously [17]. To evaluate lipid profiles, complete blood count, glucose, creatinine and INR, routine laboratory techniques were used. Fibrinogen was determined using the Clauss assay. High-sensitivity CRP was measured by immunoturbidimetry (Roche Diagnostics, Mannheim, Germany). Plasma D-dimer was measured with the Innovance D-dimer assay (Siemens, Marburg, Germany). Plasminogen activator inhibitor-1 (PAI-1) antigen (American Diagnostica, Stamford, CT, USA) was measured using an ELISA, while $\alpha_{2}$-antiplasmin $\left(\alpha_{2}\right.$ AP) and plasminogen were measured by chromogenic assays (STA Stachrom antiplasmin and STA Stachrom plasminogen, Diagnostica Stago, Asnières, France). Plasma-activated TAFI was measured by a chromogenic assay using the ACTICHROME ${ }^{\circledR}$ Plasma TAFI Activity Kit (American Diagnostica). We measured activated and inactivated TAFI (TAFIa/ai) antigen in plasma using a commercially available ELISA kit (Imubind TAFIa/ai antigen ELISA; American Diagnostica) and results were expressed as percentage of pooled plasma from healthy volunteers. Plasma FVIII activity was determined using a one-stage clotting assay (Siemens).

To evaluate clot properties and thrombin generation, venous blood samples (vol/vol, 9:1 of $3.2 \%$ trisodium citrate) were centrifuged at $2000 \times \mathrm{g}$ for $10 \mathrm{~min}$ within $30 \mathrm{~min}$ of the draw, and the supernatant was aliquoted and stored at $-80{ }^{\circ} \mathrm{C}$ until analysis. All measurements were performed by technicians blinded to the origin of the samples.

Plasma thrombogenic potential was assessed with calibrated automated thrombography (CAT) (Thrombinoscope BV, Maastricht, the Netherlands) in a 96-well plates fluorometer (Ascent Reader, Thermolab Systems OY, Helsinki, 
Finland) [18]. Fibrin clot permeation was determined by means of a pressure-driven system $[19,20]$. Based on the volume of a buffer flowing through a clot, permeability coefficient $\left(\mathrm{K}_{\mathrm{s}}\right)$ was calculated. Lysability of clots was determined by measuring clot lysis time (CLT), defined as the time from the midpoint of the clear-to-maximum-turbid transition to the midpoint of the maximum-turbid-to-clear transition, and by measuring the maximum rate of increase in D-dimer levels $\left(\mathrm{D}^{-} \mathrm{D}_{\text {rate }}\right)$ and maximum D-dimer concentrations (D-D $\mathrm{D}_{\max }$ ) during clot perfusion with $\operatorname{rtPA}[19,21,22]$.

To determine turbidity measurements, plasma citrated samples were mixed with human thrombin (Sigma-Aldrich) and $\mathrm{CaCl}_{2}$ and absorbance was read at $405 \mathrm{~nm}$ as described [23].

\section{Genotyping}

The FV Leiden, prothrombin 20210A, FXIII Val34Leu and $\alpha$-fibrinogen Thr312Ala polymorphisms were determined as described [19].

\section{Follow-up}

The patients completed a follow-up period of a minimum of 24 months since enrolment. At their last visit they were assessed using a Villalta scale in order to determine the presence and severity of PTS. Patients were diagnosed with PTS if the Villalta score was at least 5. A score of 5-9 points was categorised as mild, 10-14 as moderate, and 15 and more as severe manifestation of the disease [24].

Every 6 months participants were examined at the clinic in terms of the presence of a venous ulcer. In individuals presented with an ulcer additional data on time of its appearance, healing and recurrence were collected. The patients were examined in terms of the recurrence of DVT during follow-up. Use of compression stockings and anticoagulation treatment were also monitored.

\section{Statistical analysis}

The study was powered to have a $80 \%$ chance of detecting a $16 \%$ difference in CLT using a one-sided $P$ value of 0.025 , based on the values of clot lysis time in the previous study [17]. In order to demonstrate such a difference, or a greater one, in total 180 subjects were required, including 12 patients with ulceration.

Categorical variables are presented as numbers and percentages. Continuous variables are expressed as mean \pm standard deviation (SD) or median and interquartile range (IQR). Normality was assessed by the Shapiro-Wilk test. Equality of variances was assessed using the Levene's test. Differences between groups were compared using the Student's or the Welch's $t$ test depending on the equality of variances for normally distributed variables. The Mann-Whitney $U$-test was used for non-normally distributed continuous variables. Categorical variables were compared by the Pearson's Chi squared test or the Fisher's exact test if $20 \%$ of cells had an expected count of $<5$. Univariate and multivariate Cox proportional hazard analysis was performed to identify independent predictors of the outcomes. The multivariate models were fitted using backward stepwise regression. Variables that were associated with the occurrence of the outcomes in univariate models with a significance level of $P<0.25$ were selected for possible inclusion in the multivariate logistic regression model. The log-rank statistic was used to test the differences in the outcomes between the groups. Two-sided $P$-values $<0.05$ were considered statistically significant. All calculations were done with $\mathrm{JMP}^{\circledR}$, Version 14.2.0 (SAS Institute Inc., Cary, NC, USA).

\section{Results}

Since eleven patients were lost to follow-up, a total of 186 patients were included in the final analysis. Characteristics of the study population were shown in Table 1 .

After a median follow-up of 53 months, PTS was diagnosed in 57 subjects $(30.6 \%)$, including 21 cases of mild (11.3\%), 12 cases of moderate (5.9\%) and 24 (12.9\%) cases of severe PTS. Venous ulcers occurred in 12 (6.45\%) individuals. The presence of an ulcer on the lower extremity persisted for 6 to 17 months (10 months, median). In one case the ulcer remained unhealed until the end of observation period. Four patients $(2.15 \%)$ suffered from recurrent ulcers. Individuals who developed ulcers were more frequently obese compared to the remainder, with no other demographics and clinical intergroup differences (Table 1). Compression therapy and anticoagulant therapy were reported by most patients in the ulcer group compared with the non-ulcer group (Table 1).

Analysis of haemostatic markers showed that the occurrence of ulcers was associated with lower concentration $\alpha_{2-}$ antiplasmin and higher TAFI activity at enrolment (Table 1). There were no intergroup differences in fibrinogen, CRP and thrombin generation. The four genetic polymorphisms tested were similarly distributed in the two groups (Table 1).

As shown in Table 2, at 3 months since the DVT diagnosis, $\mathrm{K}_{\mathrm{s}}$ was $13.0 \%$ lower in the patients who developed venous ulcers during follow-up as compared to the remainder, while CLT was $17.4 \%$ longer in this group. Furthermore, we observed $13.1 \%$ longer lag phase and $5 \%$ greater $\Delta \mathrm{Abs}$ in the ulcer group compared with the non-ulcer group (Table 2). Both $\mathrm{D}-\mathrm{D}_{\max }$ and $\mathrm{D}-\mathrm{D}_{\text {rate }}$ values showed no intergroup differences. The so-called prothrombotic 
Table 1 Baseline patient characteristics

\begin{tabular}{|c|c|c|c|c|}
\hline Variables & Total cohort $(n=186)$ & $\begin{array}{l}\text { Patients with venous ulcer } \\
(\mathrm{n}=12)\end{array}$ & $\begin{array}{l}\text { Patients free of venous ulcer } \\
(n=174)\end{array}$ & $P$ \\
\hline Age (years) & $45(33-54.25)$ & $47.5(39.5-60.75)$ & $45(32-53.25)$ & 0.147 \\
\hline Male $[\mathrm{n}(\%)]$ & $101(54.3)$ & $4(33.3)$ & $97(55.8)$ & 0.132 \\
\hline Body mass index $\left(\mathrm{kg} / \mathrm{m}^{2}\right)$ & $26.85(24.9-29.2)$ & $33.75(30.6-36.05)$ & $26.65(24.88-28.93)$ & $<0.001$ \\
\hline Obesity [n (\%)] & $28(15.05)$ & $10(83.3)$ & $18(10.3)$ & $<0.001$ \\
\hline Active smoking [n (\%)] & $73(39.3)$ & $3(25)$ & $70(40.2)$ & 0.371 \\
\hline Family history of VTE [n (\%)] & $28(15.1)$ & $2(16.7)$ & $26(14.9)$ & 1.000 \\
\hline \multicolumn{5}{|l|}{ Clinical variables $[\mathrm{n}(\%)]$} \\
\hline Post-Trauma VTE & $38(20.4)$ & $0(0)$ & $38(21.8)$ & 0.130 \\
\hline Unprovoked VTE & $91(48.9)$ & $7(58.3)$ & $84(48.3)$ & 0.500 \\
\hline DVT alone & $57(30.7)$ & $3(25)$ & $54(31)$ & 0.758 \\
\hline $\mathrm{PE}+\mathrm{DVT}$ & $129(69.3)$ & $9(75)$ & $120(69)$ & \\
\hline Proximal DVT & $138(74.2)$ & $11(91.7)$ & $127(73)$ & 0.192 \\
\hline Anticoagulant therapy & $44(23.7)$ & $10(83.3)$ & $34(19.5)$ & $<0.001$ \\
\hline Compression therapy & $22(11.8)$ & $9(75)$ & $13(7.5)$ & $<0.001$ \\
\hline \multicolumn{5}{|c|}{ Laboratory investigations at enrolment } \\
\hline INR & $0.98(0.9-1.05)$ & $1.01(0.97-1.07)$ & $0.98(0.90-1.05)$ & 0.097 \\
\hline D-dimer (ng/mL) & $214(160-283)$ & $241(213-265)$ & $211(158-287)$ & 0.138 \\
\hline Fibrinogen $(\mathrm{g} / \mathrm{L})$ & $2.98(2.48-3.87)$ & $3.03(2.67-4.05)$ & $2.98(2.45-3.87)$ & 0.544 \\
\hline Creatinine $(\mu \mathrm{mol} / \mathrm{L})$ & $71.18 \pm 13.51$ & $70.92 \pm 11.05$ & $71.20 \pm 13.69$ & 0.944 \\
\hline Glucose $(\mathrm{mmol} / \mathrm{L})$ & $4.9(4.58-5.3)$ & $5.10(4.85-5.48)$ & $4.90(4.51-5.30)$ & 0.174 \\
\hline Triglycerides $(\mathrm{mmol} / \mathrm{L})$ & $1.16(0.71-1.72)$ & $1.34(0.90-1.68)$ & $1.15(0.71-1.72)$ & 0.754 \\
\hline $\mathrm{TC}(\mathrm{mmol} / \mathrm{L})$ & $5.05(4.2-5.78)$ & $4.91(4.43-5.93)$ & $5.07(4.19-5.78)$ & 0.831 \\
\hline LDL-C (mmol/L) & $3.04 \pm 0.86$ & $3.09 \pm 0.82$ & $3.04 \pm 0.87$ & 0.845 \\
\hline HDL-C (mmol/L) & $1.40(1.14-1.69)$ & $1.32(0.99-1.66)$ & $1.40(1.14-1.71)$ & 0.326 \\
\hline CRP (mg/L) & $1.70(1.02-2.34)$ & $2.64(1.16-7.45)$ & $1.61(1.02-2.31)$ & 0.059 \\
\hline Peak thrombin, nM & $233(198-305)$ & $230(188-307)$ & $233(199-305)$ & 0.544 \\
\hline Factor VIII (\%) & $124(103-142)$ & $120(111-143)$ & $124(102-142)$ & 0.912 \\
\hline PAI-1 (ng/mL) & $12.10(8.79-18.05)$ & $9.64(8.27-17.65)$ & $12.25(8.88-18.35)$ & 0.353 \\
\hline TAFI activity $(\mu \mathrm{g} / \mathrm{mL})$ & $25.55(20.20-30.30)$ & $31.41(26.12-39.67)$ & $25.15(20.14-29.97)$ & 0.004 \\
\hline TAFI antigen $(\%)$ & $100(89-110)$ & $108(96-116)$ & $100(89-110)$ & 0.066 \\
\hline Plasminogen (\%) & $107(97-120)$ & $106(91-119)$ & $107(98-120)$ & 0.392 \\
\hline$\alpha_{2}$-antiplasmin (\%) & $103(96-116)$ & $97(90-103)$ & $104(97-116)$ & 0.022 \\
\hline \multicolumn{5}{|l|}{ Genetic polymorphisms [n (\%)] } \\
\hline Factor V Leiden & $24(12.9)$ & $1(8.3)$ & $23(13.2)$ & 1.00 \\
\hline Prothrombin 20210A & $7(3.8)$ & $0(0)$ & $7(4)$ & 1.00 \\
\hline Factor XIII Val34Leu & $84(45.2)$ & $6(50)$ & $78(44.8)$ & 0.728 \\
\hline$\alpha$-fibrinogen Thr312Ala & $82(44.1)$ & $8(66.7)$ & $74(42.5)$ & 0.103 \\
\hline
\end{tabular}

Data are shown as mean \pm SD or a median (interquartile range) or number (percentage)

$V T E$ venous thromboembolism, $P T S$ post-thrombotic syndrome, $D V T$ deep vein thrombosis, $P E$ pulmonary embolism, INR international normalized ratio, $T C$ total cholesterol, $L D L-C$ low-density lipoprotein cholesterol, $H D L-C$ high-density lipoprotein cholesterol, $C R P C$-reactive protein, PAI-1 plasminogen activator inhibitor-1, and TAFI thrombin-activatable fibrinolysis inhibitor

phenotype of the fibrin clot, defined as the lower quartile of $\mathrm{K}_{\mathrm{s}}\left(\leq 6.5 \times 10^{-9} \mathrm{~cm}^{2}\right)$ and the top quartile of CLT (>100 $\mathrm{min}$ ), was associated with a 5.37 -fold [95\% confidence interval (CI) 1.3-21.5] higher risk of venous ulcers in patients with DVT during follow-up.

Univariate analysis showed that BMI, recurrent VTE, using of compression prior to ulcer, anticoagulation, CRP, $\triangle \mathrm{Abs}, \mathrm{CLT}$ and TAFI activity were positively associated with the risk of a venous leg ulcer, and shorter lag phase, $\mathrm{K}_{\mathrm{s}}$, and $\alpha_{2}$-antiplasmin increased the risk of venous ulcer occurrence (data not shown). A multivariate model showed that CLT, BMI and $\alpha_{2}$-antiplasmin are predictors of the ulcer occurrence (Supplementary Table S1). 
Table 2 Comparison of fibrin clot properties

\begin{tabular}{llllr}
\hline Variable & Total cohort $(\mathrm{n}=186)$ & $\begin{array}{l}\text { Patients with venous } \\
\text { ulcer }(\mathrm{n}=12)\end{array}$ & $\begin{array}{l}\text { Patients free of } \\
\text { venous ulcer }(\mathrm{n}=174)\end{array}$ & $P$ \\
\hline $\mathrm{K}_{\mathrm{s}}, 10^{-9} \mathrm{~cm}^{2}$ & $7.45 \pm 1.27$ & $6.53 \pm 0.45$ & $7.51 \pm 1.28$ & $<0.001$ \\
$\mathrm{CLT}(\mathrm{min})$ & $88(72-100)$ & $101(88-114)$ & $86(72-99)$ & 0.007 \\
Lag phase $(\mathrm{s})$ & $41(37-46)$ & $36.5(35-39.75)$ & $42(38-46)$ & 0.003 \\
$\Delta$ Abs $(405 \mathrm{~nm})$ & $0.81(0.75-0.86)$ & $0.84(0.81-0.9)$ & $0.8(0.74-0.86)$ & 0.03 \\
$\mathrm{D}_{-} \mathrm{D}_{\max }(\mathrm{mg} / \mathrm{L})$ & $3.99(3.60-4.39)$ & $3.68(3.59-3.99)$ & $4.07(3.6-4.39)$ & 0.132 \\
$\mathrm{D}_{-} \mathrm{D}_{\text {rate }}(\mathrm{mg} / \mathrm{L} / \mathrm{min})$ & $0.072 \pm 0.008$ & $0.072 \pm 0.009$ & $0.072 \pm 0.008$ & 0.68 \\
\hline
\end{tabular}

Values are given as mean $\pm \mathrm{SD}$ or a median (interquartile range)

$\mathrm{K}_{\mathrm{s}}$ indicates permeability coefficient; $C L T$ clot lysis time, $\triangle A b s$ maximum absorbance, $D-D_{\max }$ maximum $\mathrm{D}$-dimer levels in the lysis assay, and $D-D_{\text {rate }}$ maximum rate of increase in D-dimer levels in the lysis assay
Patients who developed PTS presented in Supplementary Table $\mathrm{S} 2$ were characterised by a $10 \%$ lower $\mathrm{K}_{\mathrm{s}}, 14 \%$ longer CLT, $9 \%$ shorter lag phase, and $4 \%$ higher $\Delta$ Abs, all typical of the prothrombotic fibrin clot phenotype (Supplementary Table S3).

\section{Discussion}

To our knowledge, this study is the first to show association of the plasma fibrin clot phenotype and venous ulcers among DVT patients. We found that denser fibrin clot formation and reduced susceptibility to lysis not only predispose to PTS, which is in line with our previous study with a shorter follow-up of 12 months [17], but also significantly increase the risk of a post-thrombotic venous ulcer. Our findings suggest that unfavourable fibrin clot properties may have a predictive value in terms of venous ulcers.

Analysis of several proteins involved in fibrinolysis in the context of post-thrombotic leg ulcers provided additional interesting observations. We noted that DVT patients with venous ulcers had higher baseline TAFI activity accompanied by lower $\alpha_{2}$-antiplasmin compared with the remaining subjects, without any differences in PAI-1 or plasminogen. The observation on TAFI is consistent with our previous study, where hypofibrinolysis was associated with higher TAFI activity in PTS patients [17]. The current finding supports involvement of elevated TAFI activity in PTS. Surprisingly, in individuals in the ulcer group we observed lower $\alpha_{2}$-antiplasmin levels, but all within the reference range, without any difference in this parameter related to the presence or absence of PTS. This key fibrinolysis inhibitor [25] accounts for about $90 \%$ of plasmin inhibition in vivo [26]. Since the resistance of thrombi to plasmin digestion depends primarily on the amount of $\alpha_{2} \mathrm{AP}$ incorporated within fibrin, lower antifibrinolytic antiplasmin, though linearly not correlated to CLT, might suggest that increased, not impaired, fibrinolysis enhances the post-thrombotic ulcer formation. However, we found that prolonged CLT is associated with this complication of DVT, therefore it is unlikely that antiplasmin-mediated impact on global efficiency of fibrinolysis explains the protective effect of lower $\alpha_{2} \mathrm{AP}$ on ulcer formation. Lower antiplasmin could indicate changes in a physiological ratio of two forms of this protein [27]. Antiplasmincleaving enzyme (APCE) cleaves Met- $\alpha_{2}$ AP to Asn- $\alpha_{2}$ AP, which is more efficiently incorporated into fibrin and makes it resistant to plasmin digestion. Another potential factor affecting the antiplasmin could be $\alpha_{2} \mathrm{AP}$ posttranslational modifications such as oxidation that may modulate the protein's function [28]. Mechanisms underlying the role of antiplasmin in the context of post-thrombotic ulcers require further research. We cannot exclude that modulation of antiplasmin activity might prevent venous ulcer formation.

Looking at several demographic and clinical factors and their associations with post-thrombotic ulcers, we identified obesity as the strongest predisposing factor, which agrees with several papers $[9,10]$. Galanaud et al. found that the male sex increases the risk of ulcers after VTE at 1 year only, whereas after 2 or 3 years of follow-up this association was no longer significant [12]. We did not observe a higher proportion of males in the ulcer group during a few years since DVT, which is similar to that study. Since diabetic patients had been excluded from our study due to prothrombotic clot alterations observed commonly in diabetes [17, 29], the impact of this disease on post-thrombotic ulcers was not evaluated in the present study. Anticoagulant therapy and the use of compression stockings did not reduce the risk of venous ulceration [12], which agrees with our findings.

We found higher CRP levels of borderline statistical significance in the ulcer group, while fibrinogen concentrations were similar. Previous studies demonstrated that elevated plasma levels of inflammatory biomarkers, including CRP, were associated with PTS [30, 31]. This study supports the view that elevated CRP is not related to the risk of leg ulceration. Nonetheless, increased CRP levels in patients with ulcers might indicate the presence of infection in affected tissues [32]. On the other hand, we confirmed that the presence of PTS has been linked to systemic inflammation. We did 
not observe any impact of heightened thrombin generation at baseline on development of venous ulcers in follow-up, therefore elevated thrombin, the well-known factor leading to more compact clot network formation [33], does not contribute to unfavourable fibrin clot properties in this patient group.

This study has several limitations. Although the study was adequately powered, the number of patients with venous ulcers was limited, however the prevalence of post-thrombotic ulcers is low as shown in other studies [34, 35]. Our study was designed to assess fibrin clots in relation to PTS in patients below 70 years, free of severe thrombophilia, cancer or diabetes to avoid factors adversely affecting clot phenotype [22, 36, 37]. Our results cannot be easily extrapolated to those patient subsets. Molecular mechanisms underlying elevated risk of post-thrombotic ulcers in the presence of the prothrombotic clot phenotype were beyond the scope of the current study. It has been shown that decreased $\mathrm{K}_{\mathrm{s}}$ not only correlates with thromboembolic events, but also is associated with an elevated bleeding risk and, consequently, with disturbances in healing of mild skin injuries [38]. This observation may explain at least in part the persistence of venous ulcers in individuals characterised by unfavourable fibrin clot phenotype.

The current study demonstrates that patients who develop venous ulcers following DVT produce dense and lysis-resistant fibrin clots. It might be speculated that measurement of clot permeability and CLT might be useful in identifying DVT patients at high risk of post-thrombotic ulcers. How to reduce this risk should be a subject of further investigation.

Acknowledgements This study was supported by Jagiellonian University Medical College (No. K/ZDS/007717, to A.U.).

\section{Compliance with ethical standards}

Conflict of interest The authors declare that they have no conflict of interest.

Ethical approval All procedures performed in studies involving human participants were in accordance with the ethical standards of the Jagiellonian University Medical College Ethical Committee and with the 1964 Helsinki declaration and its later amendments or comparable ethical standards.

Open Access This article is distributed under the terms of the Creative Commons Attribution 4.0 International License (http://creativeco mmons.org/licenses/by/4.0/), which permits unrestricted use, distribution, and reproduction in any medium, provided you give appropriate credit to the original author(s) and the source, provide a link to the Creative Commons license, and indicate if changes were made.

\section{References}

1. Kahn SR (2016) The post-thrombotic syndrome. Hematol Am Soc Hematol Educ Progr 1:413-418

2. O'Donnell TF, Passman MA, Marston WA et al (2014) Management of venous leg ulcers: clinical practice guidelines of the Society for Vascular Surgery ${ }^{\circledR}$ and the American Venous Forum. J Vasc Surg 60:3S-59S. https://doi.org/10.1016/j. jvs.2014.04.049

3. Nelzen O (2008) Prevalence of venous leg ulcer: the importance of the data collection method. Phlebolymphology 15:143-150

4. Lal BK (2015) Venous ulcers of the lower extremity: definition, epidemiology, and economic and social burdens. Semin Vasc Surg 28:3-5. https://doi.org/10.1053/j.semvascsurg.2015.05.002

5. Nelson EA, Adderley U (2016) Venous leg ulcers. BMJ. Clin Evid 2016:1-36

6. Lim CS, Baruah M, Bahia SS (2018) Diagnosis and management of venous ulcers. BMJ 20:82-85. https://doi.org/10.1177/15310 03507313218

7. Crawford JM, Lal BK, Durán WN, Pappas PJ (2017) Pathophysiology of venous ulceration. J Vasc Surg Venous Lymphat Disord 5:596-605. https://doi.org/10.1016/j.jvsv.2017.03.015

8. Abelyan G, Abrahamyan L, Yenokyan G (2018) A casecontrol study of risk factors of chronic venous ulceration in patients with varicose veins. Phlebology 33:60-67. https://doi. org/10.1177/0268355516687677

9. Robertson L, Lee AJ, Gallagher K et al (2009) Risk factors for chronic ulceration in patients with varicose veins: a case control study. J Vasc Surg 49:1490-1498. https://doi.org/10.1016/j. jvs.2009.02.237

10. Vlajinac H, Marinkovic J, Maksimovic M, Radak D (2014) Factors related to venous ulceration: a cross-sectional study. Angiology 65:824-830. https://doi.org/10.1177/0003319713508218

11. Meulendijks AM, de Vries FMC, van Dooren AA et al (2018) A systematic review on risk factors in developing a first time Venous Leg Ulcer. J Eur Acad Dermatology Venereol. 33(7):1241-1248. https://doi.org/10.1111/jdv.15343

12. Galanaud JP, Bertoletti L, Amitrano M et al (2018) Predictors of post-thrombotic ulcer after acute DVT: the RIETE registry. Thromb Haemost 118:320-328. https://doi.org/10.1160/ TH17-08-0598

13. Undas A, Ariëns RAS (2011) Fibrin clot structure and function: a role in the pathophysiology of arterial and venous thromboembolic diseases. Arterioscler Thromb Vasc Biol 31:88-99. https:// doi.org/10.1161/ATVBAHA.111.230631

14. Zabczyk M, Plens K, Wojtowicz W, Undas A (2017) Prothrombotic fibrin clot phenotype is associated with recurrent pulmonary embolism after discontinuation of anticoagulant therapy. Arterioscler Thromb Vasc Biol 37:365-373. https://doi.org/10.1161/ ATVBAHA.116.308253

15. Cieslik J, Mrozinska S, Broniatowska E, Undas A (2018) Altered plasma clot properties increase the risk of recurrent deep vein thrombosis: a cohort study. Blood 131:797-807. https://doi. org/10.1182/blood-2017-07-798306

16. Sumaya W, Wallentin L, James SK et al (2018) Fibrin clot properties independently predict adverse clinical outcome following acute coronary syndrome: a PLATO substudy. Eur Heart J 39:1078-1085. https://doi.org/10.1093/eurheartj/ehy013

17. Siudut J, Grela M, Wypasek E et al (2016) Reduced plasma fibrin clot permeability and susceptibility to lysis are associated with increased risk of postthrombotic syndrome. J Thromb Haemost 14:784-793. https://doi.org/10.1111/jth.13264

18. Pankiw-Bembenek O, Zalewski J, Goralczyk T, Undas A (2012) A history of early stent thrombosis is associated with prolonged 
clot lysis time. Thromb Haemost 107:513-520. https://doi. org/10.1160/TH11-09-0662

19. Undas A, Zawilska K, Ciesla-Dul M et al (2009) Altered fibrin clot structure/function in patients with idiopathic venous thromboembolism. Blood 114:4272-4278. https://doi.org/10.1182/blood -2009-05-222380.An

20. Mills JD, Ariëns RA, Mansfield MW, Grant PJ (2002) Altered fibrin clot structure in the healthy relatives of patients with premature coronary artery disease. Circulation 106:1938-1942. https ://doi.org/10.1161/CIR.0000033221.73082.06

21. Lisman T, De Groot PG, Meijers JCM, Rosendaal FR (2005) Reduced plasma fibrinolytic potential is a risk factor for venous thrombosis. Blood 105:1102-1105. https://doi.org/10.1182/blood -2004-08-3253

22. Celińska-Löwenhoff M, Iwaniec T, Padjas A et al (2014) Altered fibrin clot structure/function in patients with antiphospholipid syndrome: association with thrombotic manifestation. Thromb Haemost 112:287-296. https://doi.org/10.1160/ TH13-11-0980

23. Zang M, Cam G, Undas A et al (2018) Altered fibrin clot properties in advanced lung cancer: impact of chemotherapy. J Thorac Dis 10:6863-6872. https://doi.org/10.21037/jtd.2018.11.19

24. Kahn SR, Partsch H, Vedantham S et al (2009) Definition of post-thrombotic syndrome of the leg for use in clinical investigations: a recommendation for standardization. J Thromb Haemost 7:879-883. https://doi.org/10.1111/j.1538-7836.2009.03294.x

25. Coughlin PB (2005) Antiplasmin: the forgotten serpin? FEBS J 272:4852-4857. https://doi.org/10.1111/j.1742-4658.2005.04881 . $\mathrm{X}$

26. Levi M, Roem D, Kamp AM et al (1993) Assessment of the relative contribution of different protease inhibitors to the inhibition of plasmin in vivo. Thromb Haemost 70:141-146. https://doi. org $/ 10.1055 / \mathrm{s}-0038-1651570$

27. Abdul S, Leebeek FWG, Rijken DC, Uitte de Willige S (2016) Natural heterogeneity of a 2-antiplasmin: functional and clinical consequences. Blood 127:538-545. https://doi.org/10.1182/blood -2015-09-670117.plasminogen

28. Bryk AH, Konieczynska M, Rostoff P et al (2019) Plasma Protein Oxidation as a determinant of impaired fibrinolysis in type 2 diabetes. Thromb Haemost 119:213-222. https://doi. org/10.1055/s-0038-1676609

29. Dunn EJ, Hess K, Ajjan RA et al (2013) Diabetes is associated with posttranslational modifications in plasminogen resulting in reduced plasmin generation and enzyme-specific activity. Blood 122:134-142. https://doi.org/10.1182/blood-2013-04494641

30. Bouman AC, Smits JJM, Ten Cate H, Ten Cate-Hoek AJ (2012) Markers of coagulation, fibrinolysis and inflammation in relation to post-thrombotic syndrome. J Thromb Haemost 10:1532-1538. https://doi.org/10.1111/j.1538-7836.2012.04798.x

31. Miron MJ, Schulman S, Selby R et al (2014) Inflammation markers and their trajectories after deep vein thrombosis in relation to risk of post-thrombotic syndrome. J Thromb Haemost 13:398 408. https://doi.org/10.1111/jth.12814

32. Goodfield M (1988) C-reactive protein levels in venous ulceration: an indication of infection? J Am Acad Dermatol 18:1048-1052. https://doi.org/10.1016/S0190-9622(88)70103-6

33. Wolberg AS, Monroe DM, Roberts HR, Hoffman M (2003) Elevated prothrombin results in clots with an altered fiber structure: a possible mechanism of the increased thrombotic risk. Blood 101:3008-3013. https://doi.org/10.1182/blood-2002-08-2527

34. Prandoni P, Lensing AWA, Prins MH et al (2004) Below-knee elastic compression stockings to prevent the post-thrombotic syndrome. Ann Intern Med 141:249-256

35. Schulman S, Lindmarker P, Holmström M et al (2006) Postthrombotic syndrome, recurrence, and death 10 years after the first episode of venous thromboembolism treated with warfarin for 6 weeks or 6 months. J Thromb Haemost 4:734-742. https:// doi.org/10.1111/j.1538-7836.2006.01795.x

36. Gronostaj K, Richter P, Nowak W, Undas A (2013) Altered plasma fibrin clot properties in patients with digestive tract cancers: links with the increased thrombin generation. Thromb Res 131:262267. https://doi.org/10.1016/j.thromres.2012.11.033

37. Dunn EJ, Ariëns RAS, Grant PJ (2005) The influence of type 2 diabetes on fibrin structure and function. Diabetologia 48:11981206. https://doi.org/10.1007/s00125-005-1742-2

38. Drabik L, Wołkow P, Undas A (2017) Fibrin clot permeability as a predictor of stroke and bleeding in anticoagulated patients with atrial fibrillation. Stroke 48:2716-2722. https://doi.org/10.1161/ STROKEAHA.117.018143

Publisher's Note Springer Nature remains neutral with regard to jurisdictional claims in published maps and institutional affiliations. 